\title{
Time-of-flight PET data determine the attenuation sinogram up to a constant.
}

\author{
Michel Defrise ${ }^{1}$, Ahmadreza Rezaei $^{2}$, Johan Nuyts $^{3}$
}

\begin{abstract}
In positron emission tomography (PET), a quantitative reconstruction of the tracer distribution requires accurate attenuation correction. We consider situations where a direct measurement of the attenuation coefficient of the tissues is not available or unreliable, and where one attempts to estimate the attenuation sinogram directly from the emission data by exploiting the consistency conditions that must be satisfied by the non-attenuated data. We show that in time-of-flight (TOF) PET, the attenuation sinogram is determined by the emission data except for a constant and that its gradient can be estimated efficiently using a simple analytic algorithm. The stability of the method is illustrated numerically by means of a $2 \mathrm{D}$ simulation.
\end{abstract}

${ }^{1}$ Dept. of Nuclear Medicine, Vrije Universiteit Brussel, B-1090, Brussels, Belgium, mail: mdefrise@vub.ac.be,

${ }^{2}$ Dept. of Nuclear Medicine, Katholieke Universiteit Leuven, B-3000, Leuven, Belgium, mail: ahmadreza.rezaei@uzleuven.be,

${ }^{3}$ Dept. of Nuclear Medicine, Katholieke Universiteit Leuven, B-3000, Leuven, Belgium, mail: johan.nuyts@uzleuven.be. 


\section{Introduction}

In positron emission tomography (PET), an accurate quantitative reconstruction of the tracer distribution requires taking into account the attenuation of the photons by the tissues. The spatial distribution of the attenuation coefficient (the attenuation image) is usually estimated by means of a CT scan, extrapolated to the required photon energy of $511 \mathrm{keV}$, and forward projected to obtain the attenuation sinogram [1]. There are situations however where this external information about the attenuation is unavailable, incomplete, or potentially inaccurate due for instance to patient motion between the transmission scan and the emission scan or to the utilization of radiological contrast agents [2]. Despite recent progresses $[3,4,5]$, estimating the attenuation from MR data in PET-MR scanners remains complex and more prone to errors than with PET-CT scanners. In all these cases, any additional information should be exploited to improve the stability and accuracy of the reconstruction. Potential sources of additional information include known values of the attenuation coefficients in various types of tissues [6] but also the emission data themselves, which have long been known to contain significant information about the attenuation. An interesting illustration of the latter is given for two-dimensional (2D) PET by theorem 2.1 in [7]: if the tracer distribution consists of finitely many point sources, one of which is outside the convex hull of the attenuating medium, then the attenuation factors are determined in a unique way for all lines of response containing a source, i.e. for all lines of response for which the attenuation factors are needed for image reconstruction. This theorem and similar properties explain the success of statistical iterative algorithms that simultaneously estimate the tracer distribution and the attenuation image from the combined emission and transmission data. The goal of these algorithms is to supplement the poor signal-to-noise ratio in the transmission data measured with external rotating positron sources in PET scanners of the previous generations $[8,9,10,11]$.

The question then naturally arises: is it possible to estimate the attenuation correction factors using only the emission PET data ? The answer is negative: emission data do not determine the attenuation correction factors [7]. The simplest examples of non-uniqueness involve problems where the tracer distribution and the attenuating medium are both radially symmetrical. There have nevertheless been attempts to solve this problem. Natterer et al $[12,13]$ use the Helgason-Ludwig consistency conditions to estimate the coefficients of a simple parametric model of the attenuation image, such as a uniform ellipse mimicking the abdomen. Iterative methods have also been described, see e.g. [14, 15, 16], as well as methods based on discrete consistency conditions [17]. Despite some encouraging results, the problem is poorly determined and in most cases the estimated attenuation image contains artefacts, which mirror structures of the activity image (these errors are referred to as cross-talk artefacts). The poor stability of this problem also explains why PET images reconstructed without attenuation correction are not only quantitatively inaccurate, but also structurally inaccurate, missing for instance lung tumors where the increased attenuation almost exactly compensates the increased tracer uptake [18]. 
This paper deals with attenuation correction in time-of-flight (TOF) PET. Recent studies $[19,20,21,22]$ have shown that time-of-flight (TOF) reconstruction is more robust than non-TOF reconstruction when the attenuation image is not known accurately. We provide new insight into this issue, with the following results. First, by exploiting the consistency condition for the TOF Radon transform, we prove in section III that under fairly general assumptions the emission 2D TOF data determine the attenuation sinogram up to an additive constant. Secondly, section IV presents an analytical algorithm to solve this problem, and an approximate analysis of noise propagation is given in section $\mathrm{V}$. Numerical results with simulated 2D TOF data in section VI illustrate the performance of this algorithm.

\section{Time-of-flight 2D PET data}

We parametrize the 2D TOF data of a tracer distribution $f(\vec{x}=(x, y))$ as

$$
p(\phi, s, t)=\int_{-\infty}^{\infty} d l f\left(s \hat{u}^{\perp}+l \hat{u}\right) w(t-l)
$$

where $s$ and $\phi$ are the usual transaxial sinogram coordinates and $w(t)$ is the TOF profile. The unit vectors

$$
\hat{u}^{\perp}=(\cos \phi, \sin \phi) \quad \hat{u}=(-\sin \phi, \cos \phi)
$$

are respectively orthogonal and parallel to the line of response. The TOF bin $t=0$ corresponds to the mid-point of the line of response (LOR). The attenuation coefficient is denoted by $\mu(\vec{x})$ and the attenuation factors are

$$
a(\phi, s)=\exp \left\{-\int_{-\infty}^{\infty} d l \mu\left(s \hat{u}^{\perp}+l \hat{u}\right)\right\}=\exp \{-(\mathcal{R} \mu)(\phi, s)\}
$$

with $\mathcal{R}$ denoting the $2 \mathrm{D}$ Radon transform. The attenuation sinogram $(\mathcal{R} \mu)(\phi, s)$ is the Radon transform of the attenuation image $\mu(\vec{x})$. The measured data are $m(\phi, s, t)=$ $p(\phi, s, t) a(\phi, s)$.

We make the following assumptions:

(i) The TOF profile is a Gaussian with standard deviation $\sigma<\infty$,

$$
w(t)=e^{-t^{2} / 2 \sigma^{2}} / \sqrt{2 \pi} \sigma .
$$

(ii) For each measured line of response $(\phi, s)$, the TOF data are measured for all $t \in \mathbb{R}$.

(iii) The tracer distribution $f(\vec{x})$ and the attenuation coefficient $\mu(\vec{x})$ are non-negative functions with continuous first derivatives and bounded supports.

(iv) No line of response is totally attenuated, so that $a(\phi, s)>0$ for all $\phi, s$.

Define the sinogram region $\Omega$ as the set of lines of response that contain activity,

$$
\Omega=\{(\phi, s) \in[0, \pi) \times \mathbb{R} \mid m(\phi, s, t)>0\}
$$

With these assumptions, the following results hold true: 
- Theorem 1: The emission data determine the $\phi$ and the $s$ derivatives of the Radon transform $\mathcal{R} \mu$ of the attenuation image for all $(\phi, s) \in \Omega$.

- Corollary 2: The emission data determine the Radon transform $\mathcal{R} \mu$ of the attenuation image up to an additive constant within the region $\Omega$.

- Corollary 3: If the attenuation factor $a(\phi, s)$ is known for some line of response $(\phi, s) \in \Omega$, then the emission data determine in a unique way all attenuation factors within $\Omega$.

\section{Proofs}

\subsection{Proof of Theorem 1}

Consider some line of response $(\phi, s) \in \Omega$. Let $f_{1}(\vec{x})$ and $f_{2}(\vec{x})$ be two tracer distributions and $\mu_{1}(\vec{x})$ and $\mu_{2}(\vec{x})$ two attenuation images which satisfy

$$
m\left(\phi^{\prime}, s^{\prime}, t\right)=p_{1}\left(\phi^{\prime}, s^{\prime}, t\right) a_{1}\left(\phi^{\prime}, s^{\prime}\right)=p_{2}\left(\phi^{\prime}, s^{\prime}, t\right) a_{2}\left(\phi^{\prime}, s^{\prime}\right)
$$

for $\left(\phi^{\prime}, s^{\prime}\right)$ in a neighborhood of $(\phi, s)$ and $t \in \mathbb{R}$. Here $p_{j}$ and $a_{j}, j=1,2$ are defined by equations (1) and (3) with $f$ and $\mu$ replaced by $f_{j}$ and $\mu_{j}$. To prove theorem 1 , we must show that

$$
\frac{\partial\left(\mathcal{R} \mu_{2}-\mathcal{R} \mu_{1}\right)}{\partial \phi}=\frac{\partial\left(\mathcal{R} \mu_{2}-\mathcal{R} \mu_{1}\right)}{\partial s}=0 .
$$

The noise-free, attenuation corrected, 2D TOF data (1) satisfy a range condition defined by the partial differential equation ([23], see [24] for the extension to 3D TOF PET):

$$
\mathcal{D} p=t \frac{\partial p}{\partial s}+\frac{\partial p}{\partial \phi}-s \frac{\partial p}{\partial t}+\sigma^{2} \frac{\partial^{2} p}{\partial s \partial t}=0 .
$$

For completeness the proof of this property is given in appendix I. Applying the differential operator $\mathcal{D}$ to $m$, and using the fact that (8) is satisfied by $p_{1}$ and $p_{2}$ and that $a_{1}$ and $a_{2}$ are independent of the TOF variable $t$, one obtains the following identity:

$$
\mathcal{D} m=p_{1}\left(t a_{1, s}+a_{1, \phi}\right)+\sigma^{2} a_{1, s} p_{1, t}=p_{2}\left(t a_{2, s}+a_{2, \phi}\right)+\sigma^{2} a_{2, s} p_{2, t}
$$

where we omit the variables $(\phi, s, t)$ and the subscript ${ }_{, \xi}$ denotes the partial derivative w.r.t. a variable $\xi$, thus,

$$
a_{j, s}=\frac{\partial a_{j}(\phi, s)}{\partial s} \quad a_{j, \phi}=\frac{\partial a_{j}(\phi, s)}{\partial \phi} \quad p_{j, t}=\frac{\partial p_{j}(\phi, s, t)}{\partial t} \quad j=1,2 .
$$

Defining the functions

$$
q_{j}(\phi, s, t)=p_{j}(\phi, s, t) \exp \left(t^{2} / 2 \sigma^{2}\right) \quad j=1,2
$$

and their partial derivatives $q_{j, t}=\partial q_{j} / \partial t$, equation (9) can be rewritten after multiplication by $a_{2} \exp \left(t^{2} / 2 \sigma^{2}\right)>0$ as,

$$
a_{2} a_{1, \phi} q_{1}+\sigma^{2} a_{2} a_{1, s} q_{1, t}=a_{2, \phi} a_{2} q_{2}+\sigma^{2} a_{2, s} a_{2} q_{2, t} .
$$


From equation (6), $a_{2} q_{2}=a_{1} q_{1}$ and $a_{2} q_{2, t}=a_{1} q_{1, t}$ (because the functions $a_{j}(\phi, s)$ are independent of $t$ ), and therefore equation (12) becomes

$$
\left(a_{2} a_{1, \phi}-a_{1} a_{2, \phi}\right) q_{1}+\sigma^{2}\left(a_{2} a_{1, s}-a_{1} a_{2, s}\right) q_{1, t}=0
$$

or, using $a_{j}>0$ and dividing by $a_{1} a_{2}$,

$$
\alpha q_{1}+\sigma^{2} \beta q_{1, t}=0
$$

with

$$
\begin{aligned}
& \alpha=\frac{\partial \log \left(a_{1}\right)}{\partial \phi}-\frac{\partial \log \left(a_{2}\right)}{\partial \phi}=\frac{\partial\left(\mathcal{R} \mu_{2}-\mathcal{R} \mu_{1}\right)}{\partial \phi}, \\
& \beta=\frac{\partial \log \left(a_{1}\right)}{\partial s}-\frac{\partial \log \left(a_{2}\right)}{\partial s}=\frac{\partial\left(\mathcal{R} \mu_{2}-\mathcal{R} \mu_{1}\right)}{\partial s} .
\end{aligned}
$$

For the fixed line of response $(\phi, s)$ that we are considering, equation (14) is an ordinary differential equation (ODE) in $t$, which must be satisfied for all $t \in \mathbb{R}$.

We first show that $\beta=0$. For, suppose $\beta \neq 0$. Then the solution of the ODE (14) is

$$
p_{1}=\exp \left(-t^{2} / 2 \sigma^{2}\right) q_{1}=C \exp \left(-\frac{(t+\alpha / \beta)^{2}}{2 \sigma^{2}}\right)
$$

with $C>0$ because $m(\phi, s, t)>0$. But comparison with equation (1) shows that $p_{1}$ defined by equation (16) corresponds to a tracer distribution which, along the line $(\phi, s)$, is a point source at $t=-\alpha / \beta$. This can be verified by noting that the unique solution of the equation

$$
C \exp \left(-\frac{(t+\alpha / \beta)^{2}}{2 \sigma^{2}}\right)=\int_{-\infty}^{\infty} d l f_{1}\left(s \hat{u}^{\perp}+l \hat{u}\right) \frac{1}{\sqrt{2 \pi} \sigma} e^{-(t-l)^{2} / 2 \sigma^{2}}
$$

is $f_{1}\left(s \hat{u}^{\perp}+l \hat{u}\right)=\sqrt{2 \pi} \sigma C \delta(l+\alpha / \beta) \ddagger$. A point source is contradictory with the assumption that $f \in C^{1}$, and therefore we conclude that $\beta=0$. Equation (14) then reduces to $\alpha q_{1}=0$, which implies $\alpha=0$ because $q_{1}>0$. This concludes the proof of theorem 1 .

\subsection{Proof of Corollary 2}

From theorem 1, the $\phi$ and $s$ derivatives of $\mathcal{R} \mu$ are determined by the emission data for all lines of response within the domain $\Omega$ defined by equation (5). Consider two arbitrary lines of response $A=\left(\phi_{a}, s_{a}\right) \in \Omega$ and $B=\left(\phi_{b}, s_{b}\right) \in \Omega$. From the Lemma in Appendix 2, these two lines of response can always be linked by a continuous piece-wise smooth curve $\mathcal{L}_{A B}$ in the sinogram, defined parametrically by $(\phi(u), s(u)), u \in[0,1]$ with $(\phi(0), s(0))=\left(\phi_{a}, s_{a}\right)$ and $(\phi(1), s(1))=\left(\phi_{b}, s_{b}\right)$, and such that $(\phi(u), s(u)) \in \Omega$ for $0 \leq u \leq 1$. Integrating equation (7) along $\mathcal{L}_{A B}$ yields

$$
\mathcal{R} \mu_{2}(\phi, s)-\mathcal{R} \mu_{1}(\phi, s)=K
$$

for $(\phi, s) \in \Omega$ and for some constant $K$, or equivalently $a_{2}(\phi, s)=e^{-K} a_{1}(\phi, s)$. This concludes the proof of Corollary 2. Corollary 3 immediately follows.

$\ddagger$ This can be seen by taking the 1D Fourier transform w.r.t. $t$. Note that since $\alpha$ and $\beta$ both change sign when swapping the indices 1 and $2, f_{2}$ is also a point source along that line, at the same location but possibly with another intensity $C$. 


\subsection{Remarks}

(i) Theorem 1 and the two corollaries allow estimating the attenuation factors $a(\phi, s)$ only within $\Omega$, the interior of the support of the emission sinogram, but this is all that is needed to reconstruct the tracer distribution $f(\vec{x})$. On the other hand, when the support of the attenuating medium is larger than the support of $f(\vec{x})$, reconstructing the attenuation image $\mu(\vec{x})$ is impossible unless additional information is available such as information over the patient bed.

(ii) The third hypothesis for Theorem 1 limits its applicability to functions $f$ and $\mu$ with continuous first derivatives. This condition is not satisfied in general but the PET detectors act as low pass filters and the data are therefore samples of a smoothed function $p$. This low-pass filtering is shift variant and it is unlikely that it would preserve the consistency condition. However, the major limitation to the practical accuracy of the method is probably related to the discrete data sampling and to the noise rather than to this differentiability condition.

(iii) The condition $m(\phi, s, t)>0$ defining the region $\Omega$ where the sinogram gradient can be recovered becomes in practice $m(\phi, s, t)>\epsilon$, with $\epsilon$ some lower bound on the activity along a line of response. Therefore, stability problems are expected for lines of response close to the boundary of the support of the sinogram. This problem will be illustrated in section VI.

(iv) According to Corollary 2, the attenuation sinogram is determined by the emission data only up to an additive constant $K$. Note however, that if the support of the tracer distribution and of the attenuation image are both the disk of radius $R$, the constant sinogram

$$
\left(\mathcal{R} \mu_{K}\right)(\phi, s)=\left\{\begin{array}{cc}
K & |s| \leq R \\
0 & |s|>R
\end{array}\right.
$$

corresponds to an attenuation image

$$
\mu_{K}(\vec{x})=\left\{\begin{array}{cc}
\frac{K}{\pi \sqrt{R^{2}-|\vec{x}|^{2}}} & |\vec{x}| \leq R \\
0 & |\vec{x}|>R
\end{array}\right.
$$

This attenuation image is singular at the boundary of the disk and hence does not satisfy assumption (iii) in section II. It is likely that an iterative algorithm including a smoothness constraint or an upper bound on the attenuation coefficient would not reach this solution.

(v) One way to apply Corollary 3 is to add outside the convex hull of the scanned object a small reference object with known attenuation and activity [25]. The attenuation $a(s, \phi)$ is then known for any LOR that crosses this reference but does not cross the scanned object, and Corollary 3 then allows recovering $a(s, \phi)$ for all LORs in $\Omega$.

(vi) The proof of Theorem 1 can be extended to the case of 3D TOF-PET with a cylindrical scanner. Only a sketch of this extension is given here. Consider a cylindrical scanner 
of length $L$ with axis along the vertical axis $z$, and two LORs $A$ and $B$ which have activity. The LOR $A$ intersects the detector area (the lateral surface of the cylinder) in two detector points with coordinates $\left(x_{A}^{1}, y_{A}^{1}, z_{A}^{1}\right)$ and $\left(x_{A}^{2}, y_{A}^{2}, z_{A}^{2}\right)$ with $\left|z_{A}^{1}\right| \leq L / 2$, $\left|z_{A}^{2}\right| \leq L / 2$. Similarly for $B$. The two LORs can be related continuously as follows:

(a) Applying Theorem 1 within the vertical plane (i.e. parallel to the z-axis) containing $A$, move continuously from $A$ to its projection $A_{p}$ onto the transaxial plane $z=0$. $A_{p}$ is the LOR linking the two detector points $\left(x_{A}^{1}, y_{A}^{1}, 0\right)$ and $\left(x_{A}^{2}, y_{A}^{2}, 0\right)$.

(b) Applying Theorem 1 within the transaxial plane $z=0$, move continuously from $A_{p}$ to the projection $B_{p}$ of LOR $B$ onto the transaxial plane $z=0 . B_{p}$ links the two detector points $\left(x_{B}^{1}, y_{B}^{1}, 0\right)$ and $\left(x_{B}^{2}, y_{B}^{2}, 0\right)$. See the lemma in appendix 2 .

(c) Applying Theorem 1 within the vertical plane containing $B$, move continuously from $B_{p}$ to $B$.

One easily checks that each step can be achieved involving only intermediate LORs that connect detectors with $|z| \leq L / 2$, thus belonging to the active area of the cylindrical scanner. For most realistic configurations, all these intermediate LORs have activity and in that case one therefore concludes that the 3D TOF-PET emission data determine, up to a single global constant, the integral of the attenuation image $\mu$ along all LORs which have activity. Due to the axial truncation of the scanner, the lemma of appendix 2 cannot be extended to 3D, and for some non simply connected activity images, several constants may be needed.

\section{Analytic inversion}

This section describes an analytic algorithm to estimate the gradient of the attenuation sinogram from 2D emission TOF data, following essentially the logic of the proof of theorem 1. Using the same notations, consider some line of response $(\phi, s) \in \Omega$. The attenuation corrected data $m / a$ are consistent and must therefore satisfy equation (8). Noting that $a(\phi, s) \neq 0$ and replacing $p$ by $m / a$, equation (8) becomes

$$
\begin{aligned}
0=\mathcal{D} & \left(\frac{m(\phi, s, t)}{a(s, \phi)}\right)=\frac{1}{a(s, \phi)} \mathcal{D} m(\phi, s, t)-\frac{m(\phi, s, t)}{a(s, \phi)^{2}}\left(t \frac{\partial a(\phi, s)}{\partial s}+\frac{\partial a(\phi, s)}{\partial \phi}\right) \\
& -\frac{\sigma^{2}}{a(s, \phi)^{2}} \frac{\partial m(\phi, s, t)}{\partial t} \frac{\partial a(\phi, s)}{\partial s}
\end{aligned}
$$

Multiplying this equation by $a(s, \phi)$, we obtain

$$
\begin{aligned}
& t \frac{\partial m(\phi, s, t)}{\partial s}+\frac{\partial m(\phi, s, t)}{\partial \phi}-s \frac{\partial m(\phi, s, t)}{\partial t}+\sigma^{2} \frac{\partial^{2} m(\phi, s, t)}{\partial s \partial t} \\
= & m(\phi, s, t)\left\{t \frac{\partial \log a(\phi, s)}{\partial s}+\frac{\partial \log a(\phi, s)}{\partial \phi}\right\}+\sigma^{2} \frac{\partial m(\phi, s, t)}{\partial t} \frac{\partial \log a(\phi, s)}{\partial s} .
\end{aligned}
$$

All factors related to $m$ can be obtained from the data, and therefore we can estimate the two quantities $\partial \log a(\phi, s) / \partial s=-\partial(\mathcal{R} \mu)(\phi, s) / \partial s$ and $\partial \log a(\phi, s) / \partial \phi=-\partial(\mathcal{R} \mu)(\phi, s) / \partial \phi$ (see equation (3)) by means of a least-square fitting in $t$. In practice this fit is done using 
the measured TOF interval $\tau=\left[t_{1}, t_{2}\right]$, which should be large enough relative to the object diameter to ensure good stability. Define (the variables $\phi, s$ are omitted)

$$
\begin{aligned}
& H_{s s}=\int_{\tau} d t\left(m t+\sigma^{2} m_{, t}\right)^{2} \quad, \quad H_{s \phi}=\int_{\tau} d t m\left(m t+\sigma^{2} m_{, t}\right) \quad, \quad H_{\phi \phi}=\int_{\tau} d t m^{2}, \\
& J_{s}=\int_{\tau} d t(\mathcal{D} m)\left(m t+\sigma^{2} m_{, t}\right) \quad, \quad J_{\phi}=\int_{\tau} d t(\mathcal{D} m) m .
\end{aligned}
$$

Then the unweighted least-square estimate of the derivatives are

$$
\frac{\partial(\mathcal{R} \mu)(\phi, s)}{\partial s}=-\frac{J_{s} H_{\phi \phi}-J_{\phi} H_{s \phi}}{H_{s s} H_{\phi \phi}-H_{s \phi}^{2}} \quad, \quad \frac{\partial(\mathcal{R} \mu)(\phi, s)}{\partial \phi}=-\frac{J_{\phi} H_{s s}-J_{s} H_{s \phi}}{H_{s s} H_{\phi \phi}-H_{s \phi}^{2}} .
$$

By Schwarz inequality, the denominator $H_{s s} H_{\phi \phi}-H_{s \phi}^{2}$ is non-negative and is zero only if $m=C\left(m t+\sigma^{2} m_{, t}\right)$ for some constant $C$ independent of $t$ (but possibly depending on $\phi$ and $s$ ). When this happens, one easily checks from equation (23) that the numerators in (24) also vanish. This $0 / 0$ undeterminacy only occurs if $f$ is a point source along this line $\S$. Therefore, equation (24) can always be calculated except if the line of response only contains a point source.

Using equation (24) for all lines of response $(\phi, s) \in \Omega$, we obtain the $s$ and the $\phi$ derivatives of the attenuation sinogram $\mathcal{R} \mu(\phi, s)$. We are then left with the problem of estimating a function $\mathcal{R} \mu(\phi, s)$ from a noisy measurement of its gradient $(\nabla \mathcal{R} \mu)(\phi, s)$ over a domain $(\phi, s) \in \Omega$, up to an additive constant. In section VI, we use a straightforward discretization of this problem with a two-point finite difference approximation of each component of the gradient. This leads to a set of linear equations with a band-diagonal matrix, which is solved with a simple linear iterative algorithm. Alternative methods exist, see e.g. [26].

Some insight into the meaning of equation (22) can be gained by noting that the whole problem is invariant for translation, hence we can consider the central time bin of a LOR through the origin of the coordinate system, thus setting $t=s=0$. The equation reduces then to

$$
\frac{\partial m(\phi, 0,0)}{\partial \phi}=m(\phi, 0,0) \frac{\partial \log a(\phi, 0)}{\partial \phi}+O\left(\sigma^{2}\right)
$$

This equation reflects the fact that, in the limit of a small time resolution $\sigma \rightarrow 0$, the data is simply equal to $m(\phi, 0,0)=f(0,0) a(0, \phi)$ and therefore in this limit the angular variation of the data is equal to the angular variation of the attenuation. The terms in $\sigma^{2}$ in equation (22) provide the correction that takes into account the finite width of the TOF profile.

\section{Approximate error analysis}

The expressions (24) for the radial and azimuthal derivatives of the attenuation sinogram are non-linear functions of the TOF data $m(\phi, s, t)$, and an exact analysis of the stability $\S$ This can be seen from equation (17) in the previous section. Note also that in the limit $\sigma \rightarrow \infty$, any distribution is essentially a point source relative to the TOF resolution, and in this case undeterminacy also results. 
is impractical. We derive an approximate variance estimate by assuming that the major contribution to the variance is due to the error on $\mathcal{D} m(\phi, s, t)$. This approximation is justified by the fact that $\mathcal{D} m(\phi, s, t)$ depends on a second derivative of the data, whereas $H_{s s}, H_{s \phi}$ and $H_{\phi \phi}$ in equation (23) only involve first derivatives of $m$. With this approximation, we only need to consider the variance on $J_{s}$ and $J_{\phi}$. Assuming that the samples of $\mathcal{D} m(\phi, s, t)$ are independent random variables with variance $\epsilon^{2}$ and that the integrals over $t$ are discretized with a TOF sampling step $\Delta_{t}$, one has:

$$
\begin{aligned}
\operatorname{Var}\left(\frac{\partial(\mathcal{R} \mu)(\phi, s)}{\partial s}\right) & \simeq \frac{\Delta_{t}}{\left(H_{s s} H_{\phi \phi}-H_{s \phi}^{2}\right)^{2}} \int_{\tau} d t \epsilon^{2}\left(\left(m t+\sigma^{2} m_{, t}\right) H_{\phi \phi}-m H_{s \phi}\right)^{2} \\
& \simeq \frac{\Delta_{t} \epsilon^{2}}{\left(H_{s s} H_{\phi \phi}-H_{s \phi}^{2}\right)^{2}}\left(H_{s s} H_{\phi \phi}^{2}-2 H_{s \phi} H_{\phi \phi} H_{s \phi}+H_{s \phi}^{2} H_{\phi \phi}\right) \\
& \simeq \frac{\Delta_{t} \epsilon^{2} H_{\phi \phi}}{H_{s s} H_{\phi \phi}-H_{s \phi}^{2}}
\end{aligned}
$$

and similarly

$$
\operatorname{Var}\left(\frac{\partial(\mathcal{R} \mu)(\phi, s)}{\partial \phi}\right) \simeq \frac{\Delta_{t} \epsilon^{2} H_{s s}}{H_{s s} H_{\phi \phi}-H_{s \phi}^{2}}
$$

Recall that $\epsilon$ represents the noise on $\mathcal{D} m(\phi, s, t)$, which is expected to be larger than the noise on the measured data $m$ since $\mathcal{D}$ involves derivatives. Nevertheless, equations (26) and (27) suggest that the derivatives of the attenuation sinogram can be recovered from the emission TOF data with a good stability for LORs such that $H_{s s} H_{\phi \phi}-H_{s \phi}^{2}$ is not too small.

To gain additional insight, consider a line of response along which the activity distribution is a centred Gaussian with standard deviation $\sigma_{f}$ and total activity equal to 1 . Using equation (1) and (4) the data is (we omit the $\phi$ and $s$ variables):

$$
m(t)=\frac{\exp \left(-t^{2} /\left(2\left(\sigma^{2}+\sigma_{f}^{2}\right)\right)\right)}{\sqrt{2 \pi} \sqrt{\sigma_{f}^{2}+\sigma^{2}}} a
$$

where $a=a(\phi, s)=\exp \{-(\mathcal{R} \mu)(\phi, s)\}$, and hence

$$
m(t) t+\sigma^{2} m_{, t}(t)=\frac{t \sigma_{f}^{2} \exp \left(-t^{2} /\left(2\left(\sigma^{2}+\sigma_{f}^{2}\right)\right)\right)}{\sqrt{2 \pi}\left(\sigma_{f}^{2}+\sigma^{2}\right)^{3 / 2}} a .
$$

Inserting this in (23) and integrating over $\tau=(-\infty,+\infty)$ yields

$$
H_{s s}=\frac{\sigma_{f}^{4}}{4 \sqrt{\pi}\left(\sigma_{f}^{2}+\sigma^{2}\right)^{3 / 2}} a^{2} \quad, \quad H_{s \phi}=0 \quad ; \quad H_{\phi \phi}=\frac{1}{2 \sqrt{\pi}\left(\sigma_{f}^{2}+\sigma^{2}\right)^{1 / 2}} a^{2}
$$

and from (26),

$$
\operatorname{Var}\left(\frac{\partial(\mathcal{R} \mu)(\phi, s)}{\partial s}\right) \simeq \frac{4 \Delta_{t} \epsilon^{2} \sqrt{\pi}\left(\sigma_{f}^{2}+\sigma^{2}\right)^{3 / 2}}{\sigma_{f}^{4}} a^{-2}
$$

For this simple example the variance on the radial derivative of the attenuation sinogram increases as $\sigma^{3}$ when the TOF resolution is larger than the object size $\left(\sigma>>\sigma_{f}\right)$, and decreases down to a constant when $\sigma<<\sigma_{f}$. In the limit of a point like object $\sigma_{f} \rightarrow 0$, the variance becomes infinite as expected from the proof in section III. 


\section{Numerical example}

A 2D TOF simulation experiment was performed to illustrate the method and to obtain a first assessment of its stability to noise. In this experiment, the gradient of the attenuation sinogram $\mathcal{R} \mu$ is estimated from the TOF emission data using equation (24). Several methods could be used to estimate the attenuation sinogram from its gradient. In this study, we have used the iterative Landweber algorithm. Finally, to determine the additive constant (see equation (18)), we assume that the image can be segmented to impose the known attenuation coefficient to a region containing (mostly) tissue.

\subsection{The software phantom}

Figure 1 shows the true activity and attenuation distributions. The diameter of the field of view was $40 \mathrm{~cm}$. The TOF-resolution profile was Gaussian with a full-width at half maximum (FWHM) of 500 ps, corresponding to a spatial FWHM of $7.5 \mathrm{~cm}$. The attenuation
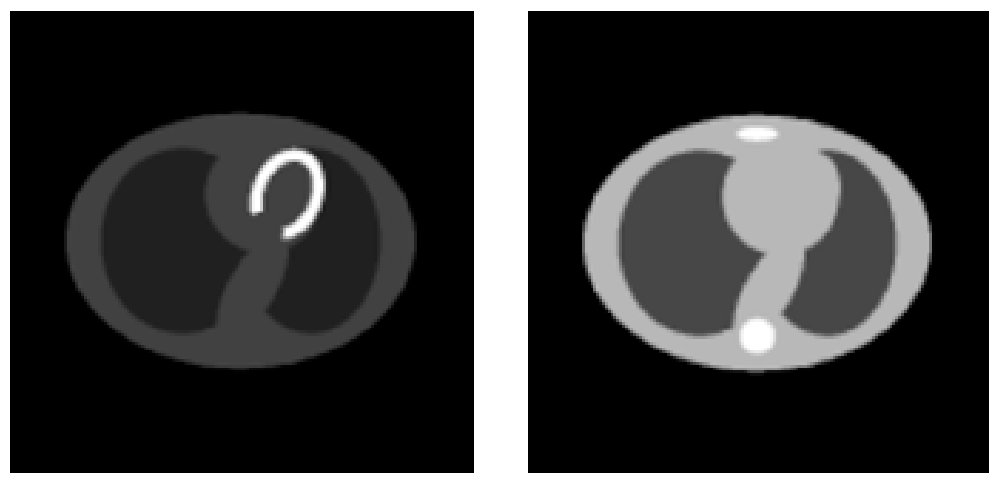

Figure 1. Activity $f$ (left) and attenuation $\mu$ (right) images of the simulated phantom. The image size is $40 \mathrm{~cm} \times 40 \mathrm{~cm}$, the simulated TOF resolution was $7.5 \mathrm{~cm}$ FWHM, corresponding to $500 \mathrm{ps}$.

coefficients were $0.095 / \mathrm{cm}$ for tissue, $0.0317 / \mathrm{cm}$ for lung, $0.142 / \mathrm{cm}$ for bone and $0 / \mathrm{cm}$ for air. To generate the data, the activity and the attenuation images were sampled on a $384 \times$ 384 grid (pixel size of $0.104 \mathrm{~cm}$ ), and forward projected to obtain sinograms of 128 angular samples over $180^{\circ}, 384$ uniform radial samples and 128 TOF-bins. The attenuation image was forward-projected using Joseph's method. The activity image was forward-projected with a TOF-PET projector implemented by rotating the image for each angle $\phi$ such that the LORs are parallel to the columns, and then convolving the image with the 1D Gaussian TOF-kernel.

Prior to estimation of the attenuation sinogram, the simulated TOF data were rebinned to $128 \times 128 \times 128$ bins with pixel size $\Delta_{\phi}=1.4^{\circ}$ along $\phi, \Delta_{s}=0.3125 \mathrm{~cm}$ along $s$ and $\Delta_{t}=0.3125 \mathrm{~cm}$ along $t$. The attenuation sinogram was reconstructed into a $128 \times 128$ image grid with pixel size of $0.3125 \mathrm{~cm}$. 


\subsection{Estimating the gradient of the attenuation sinogram}

We observed that noise propagation can be strongly reduced by a moderate smoothing of the attenuated emission sinogram in three dimensions. The noise-free and the noisy data were all smoothed with a Gaussian kernel of 2 pixels FWHM along $s(0.625 \mathrm{~cm})$ and along $\phi\left(2.8^{\circ}\right)$ and 0.7 times the TOF-FWHM along $t(5.25 \mathrm{~cm})$. This latter smoothing was taken into account by increasing the value of $\sigma$ accordingly in the subsequent calculations, as $\sigma^{2}=\sigma_{T O F}^{2}+\left(0.7 \times \sigma_{T O F}\right)^{2}$. The derivatives along $\phi, s$ and $t$ were approximated as finite differences, and the integrals over $t$ in equation (23) were approximated as a Riemann sum. Straightforward implementation of (24) then yields estimates of the derivatives of the attenuation sinogram. Results for a simulation without and with noise are shown in figures 2 and 3 respectively. For the simulation with noise, the highest expected count (Poisson mean) was 3.7 photons in the attenuated TOF-sinogram, and in the corresponding TOFintegrated sinogram (i.e. the non-TOF sinogram shown in figure 3) the maximum expected count was 132 photons.

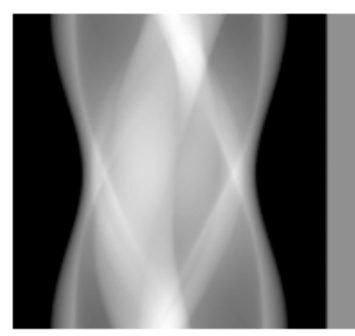

$(\mathrm{R} \mu)(\phi, \mathrm{s})$

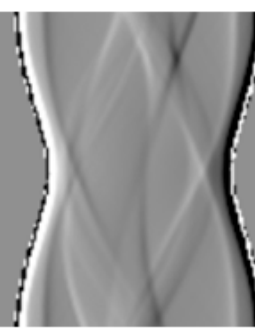

$d(R \mu) / d s$ estim

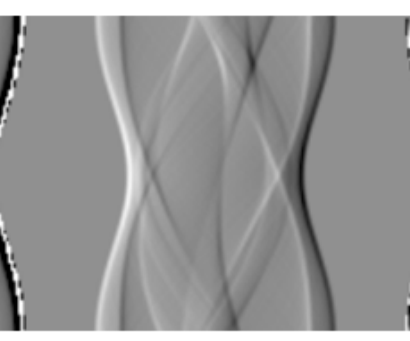

$d(R \mu) / d s$ true

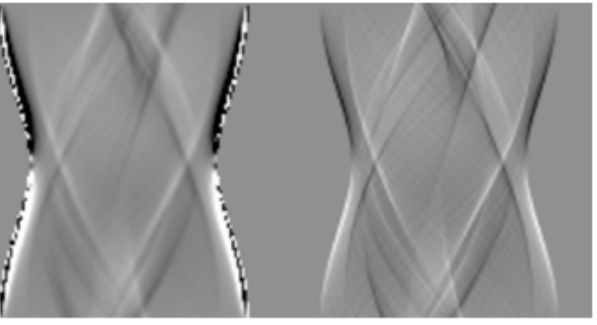

$\mathrm{d}(\mathrm{R} \mu) / \mathrm{d} \phi$ estim
$\mathrm{d}(\mathrm{R} \mu) / \mathrm{d} \phi$ true

Figure 2. Reconstruction from noise free TOF emission data. The attenuation sinogram $\mathcal{R} \mu$ is shown at the left. The subsequent images show the estimated and true radial and angular derivatives of $\mathcal{R} \mu$.
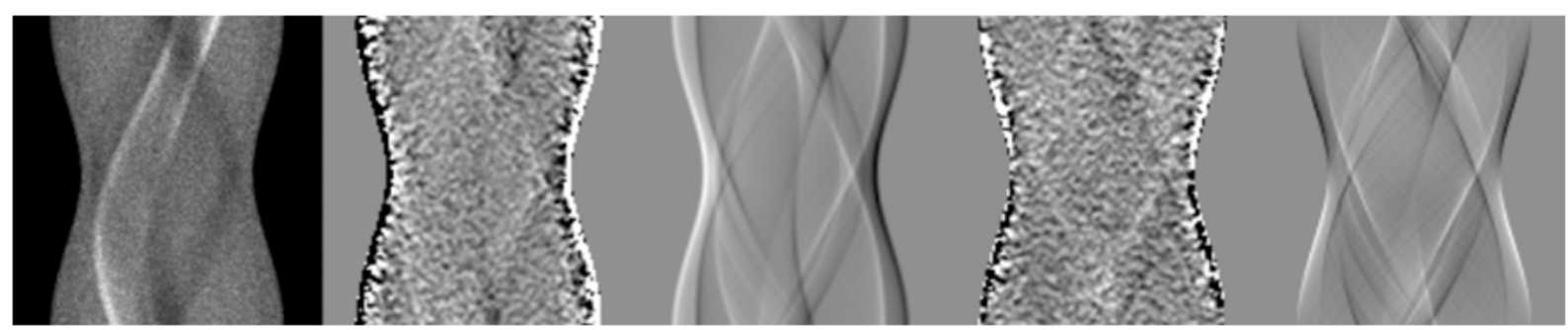

$\int m(\phi, s, t) d t$

$d(R \mu) / d s$ estim

$\mathrm{d}(\mathrm{R} \mu) / \mathrm{ds}$ true

$d(R \mu) / d \phi$ estim

$\mathrm{d}(\mathrm{R} \mu) / \mathrm{d} \phi$ true

Figure 3. Reconstruction from noisy TOF emission data. The TOF-integrated noisy emission sinogram is shown at the left. The subsequent images show the estimated and true radial and angular derivatives of $\mathcal{R} \mu$. 


\subsection{Estimating the attenuation sinogram from its gradient}

As illustrated in figure 2 and 3, the estimates of the derivatives are inaccurate near the boundaries of the object, even in the absence of noise. This is expected from the discussion in the previous sections, because these LORs have vanishing activity as one approaches the boundary. To identify these unreliable estimates, the maximum of the absolute values is computed in a central region of the derivative sinograms. All pixels with an absolute value exceeding this maximum are excluded from the subsequent calculations, together with their 4 nearest neighbors along the same row $\phi$. This heuristic approach eliminated most of the unreliable values from contributing to the final estimate. For the example in figure 2 and 3 (2nd and 4th columns), the excluded pixels correspond to the totally white and the totally black pixels, which are all located close to the boundaries of the sinogram.

An iterative Landweber algorithm is applied to estimate the attenuation sinogram $\mathcal{R} \mu$ from its derivatives. We denote by $R_{s}$ and $R_{\phi}$ the sampled $128 \times 128$ derivative sinograms estimated previously based on (24) and rescaled by the corresponding sampling intervals so that $R_{s} \simeq \Delta_{s} \partial \mathcal{R} \mu / \partial s$ and $R_{\phi} \simeq \Delta_{\phi} \partial \mathcal{R} \mu / \partial \phi$. We denote by $S^{k}$ the estimate of the attenuation sinogram $\mathcal{R} \mu$ at the $\mathrm{k}^{\text {th }}$ iteration. The algorithm updates the estimate as

$$
\begin{aligned}
& S^{0}=0 \\
& S^{k+1}=S^{k}+\omega \alpha_{s} M_{s} D_{s}^{*}\left(R_{s}-D_{s} S^{k}\right)+\omega \alpha_{\phi} M_{\phi} D_{\phi}^{*}\left(R_{\phi}-D_{\phi} S^{k}\right)
\end{aligned}
$$

where $D_{\xi}$ is the discrete approximation of the derivative with respect to $\xi=s, \phi$. We implemented $D_{\xi}$ as a convolution with $[-1 / 2,0,1 / 2]$ along $\xi=s, \phi$. The adjoint operator $D_{\xi}^{*}$ is the convolution with $[1 / 2,0,-1 / 2]$ along $\xi$. The diagonal matrix $M_{s}$ selects the reliable pixels from $R_{s}$ as described above, and similar for $M_{\phi}$. Finally, the parameters $\alpha_{s}>0$ and $\alpha_{\phi}>0$ define the relative weight given to the radial and azimuthal derivatives, and $\omega$ is a relaxation factor. To ensure convergence of the Landweber iteration [27], the relaxation factor should be in the interval $(0,2 / L)$, where $L$ is the largest eigenvalue of the matrix $A=\alpha_{s} D_{s}^{*} D_{s}+\alpha_{\phi} D_{\phi}^{*} D_{\phi}$. Ignoring subtleties near the image boundary, this matrix is circulant and corresponds to a $2 \mathrm{D}$ convolution with the kernel

$$
\left(\begin{array}{ccccccccc}
\ldots & 0 & 0 & 0 & 0 & 0 & 0 & 0 & \ldots \\
\ldots & 0 & 0 & 0 & -\alpha_{\phi} / 4 & 0 & 0 & 0 & \ldots \\
\ldots & 0 & 0 & 0 & 0 & 0 & 0 & 0 & \ldots \\
\ldots & 0 & -\alpha_{s} / 4 & 0 & \alpha_{s} / 2+\alpha_{\phi} / 2 & 0 & -\alpha_{s} / 4 & 0 & \ldots \\
\ldots & 0 & 0 & 0 & 0 & 0 & 0 & 0 & \ldots \\
\ldots & 0 & 0 & 0 & -\alpha_{\phi} / 4 & 0 & 0 & 0 & \ldots \\
\ldots & 0 & 0 & 0 & 0 & 0 & 0 & 0 & \ldots
\end{array}\right)
$$

The eigenvalues of a circulant $n \times n$ matrix with elements $c_{k}$ are given by [28]:

$$
\text { eigenvalue }_{m}=\sum_{k} c_{k} e^{-2 \pi i m k / n}, \quad \text { with } i=\sqrt{-1}, \quad m=0, \ldots, n-1
$$

It follows that the magnitude of the largest eigenvalue of $D_{s}^{*} D_{s}$ and of $D_{\phi}^{*} D_{\phi}$ does not exceed 1 , therefore the largest eigenvalue of $A$ does not exceed $\alpha_{s}+\alpha_{\phi}$. The results shown in this paper were obtained with 5000 iterations, $\alpha_{s}=\alpha_{\phi}=1$ and $\omega=1 / 2$. 
The estimated sinogram $S$ has an arbitrary offset $K$, which can only be estimated if prior knowledge is available. We assume here that a first reconstruction $F_{S}^{0}$ is made, that $F_{S}^{0}$ can be segmented, and that one has some prior knowledge about the attenuation coefficient(s) in some of the segmented regions. The value of $K$ can then be estimated by imposing the known attenuation coefficient(s) (for instance in a least squares sense). Here this was done by using a unit non-TOF sinogram $S_{1}$ (which is set to 1 for all LORs that have some activity in the original sinogram and zero elsewhere) and its reconstruction $F_{1}$. The offset $K$ is then obtained by requiring that the image $F_{S}^{0}+K F_{1}$ satisfies the prior knowledge about the attenuation.

In this experiment, we used as prior knowledge the attenuation coefficient of tissue and used simple thresholding to obtain a region containing mostly tissue and bone. We forced the median value of that region to the attenuation of tissue. The reconstructions were made with filtered backprojection. These reconstructions are less accurate closer to the object boundary, because of the increasing contribution of the unreliable boundary values during application of the ramp filter. Attenuation sinograms estimated with this approach are shown in figure 4 for simulations without noise, with moderate noise and with high noise. The first two correspond to the results shown in figures 2 and 3 respectively. In the last simulation, the maximum noise-free count in the TOF-sinogram was 1.23 photons. The maximum in the corresponding non-TOF sinogram was 44.1 photons. Figure 5 shows a horizontal profile through the four sinograms of figure 4 . Note that estimating $K$ from the reconstructions created some bias in the sinograms, because of the reconstruction errors near the boundary of the object.

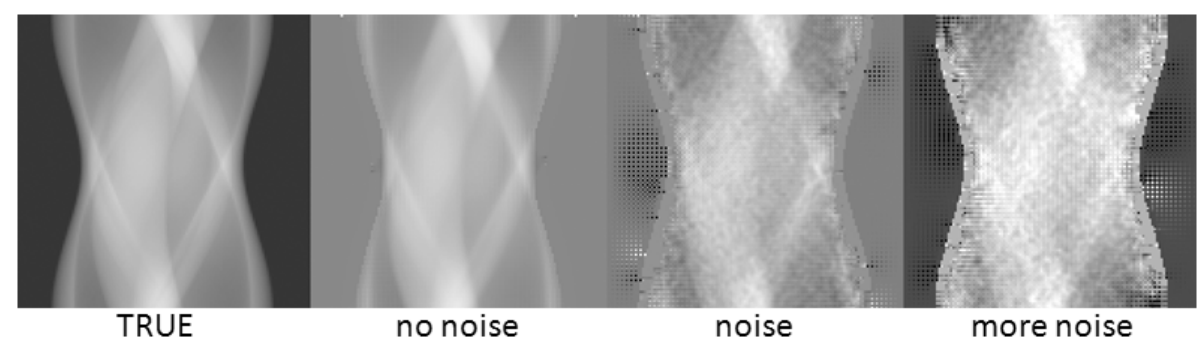

Figure 4. The true and estimated attenuation sinograms for the cases with no, moderate and high noise.

We also present in figure 6 the reconstructions of the attenuation image to illustrate the performance of the method. However, as mentioned before, no reconstruction of the attenuation image is needed for PET attenuation correction. For comparison, reference images are shown as well, which were obtained from the attenuated emission sinogram by assuming that the true activity distribution (figure 1) was known. In that case, the reconstruction problem reduces to that in transmission tomography. These reference CT reconstructions were made with filtered backprojection, and with a maximum-likelihood algorithm (MLTR, [6]) dedicated to transmission tomography. We have applied smoothing to the two FBP and MLTR reference images to obtain a resolution similar to that obtained 


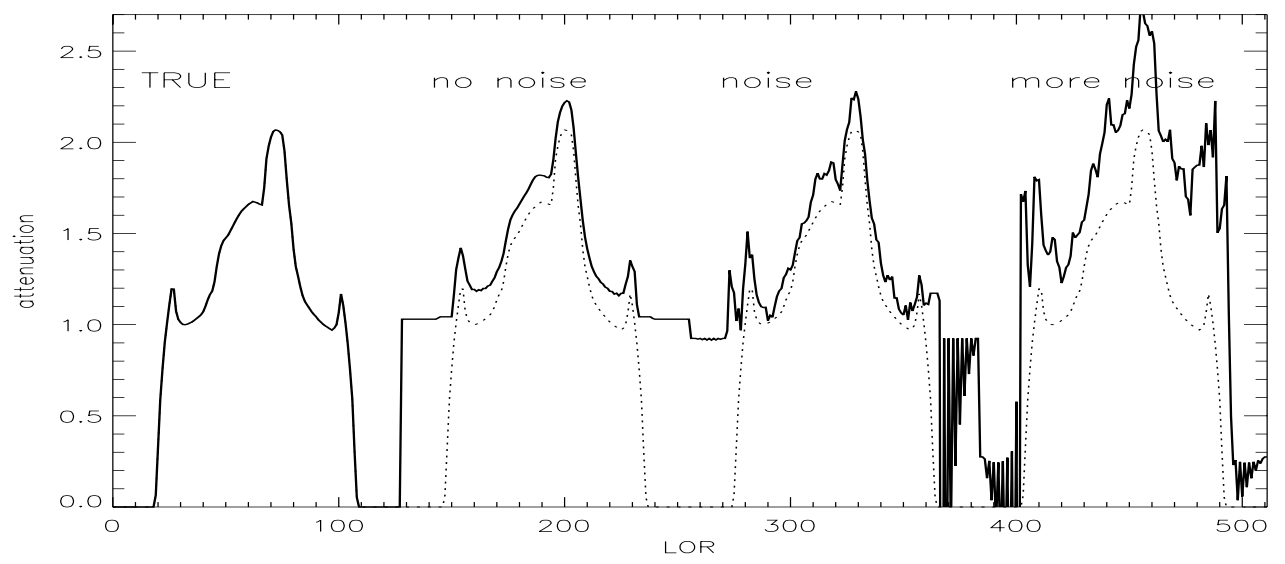

Figure 5. Horizontal profiles through the sinograms shown in figure 4 (mean of 6 rows around row 25 (of 128)). The dashed curve is the true profile, the solid curves show the true profile and the profiles through the noise-free and two noisy sinograms.

with the new algorithm.

Small residual cross-talk from the activity in the heart is visible in the three noisefree reconstructions of the attenuation image in figure 6 , this is tentatively attributed to the use of different projectors for simulation and reconstruction. Figure 6 also shows that the accuracy of the reconstructions is lower in the neighbourhood of the phantom external boundary. This is true even for the two reference CT reconstructions because they use as "blank" scan the (assumed known) unattenuated emission sinogram, which has low values in the LORs tangent to the phantom boundary.

\section{Conclusion}

The main contribution of this paper is to show that when time-of-flight information is available, the joint estimation of attenuation and activity from a PET emission sinogram has a unique solution, except for a constant. The proof of this property suggested an analytical method to estimate the attenuation sinogram, which is local in the sense that the derivatives of the attenuation sinogram for a given line of response are calculated using only neighboring lines of response. The implementation of the analytic method for simulated data demonstrates that Theorem 1 is not only a theoretical result but has a practical impact.

As expected, equation (31) shows that the variance on the estimated derivative sinograms reaches its asymptotic minimal value for perfect TOF resolution. However, at least for a Gaussian object, the variance is only $10 \%$ higher than this asymptotic value when the width of the object is 4 times larger than the width of the TOF kernel. That indicates that with current state of the art TOF systems, good results are expected for clinical applications.

How to optimally extract the information from the time-of-flight data is still an open question. The analytic method was applied here for illustration and validation, but it is 

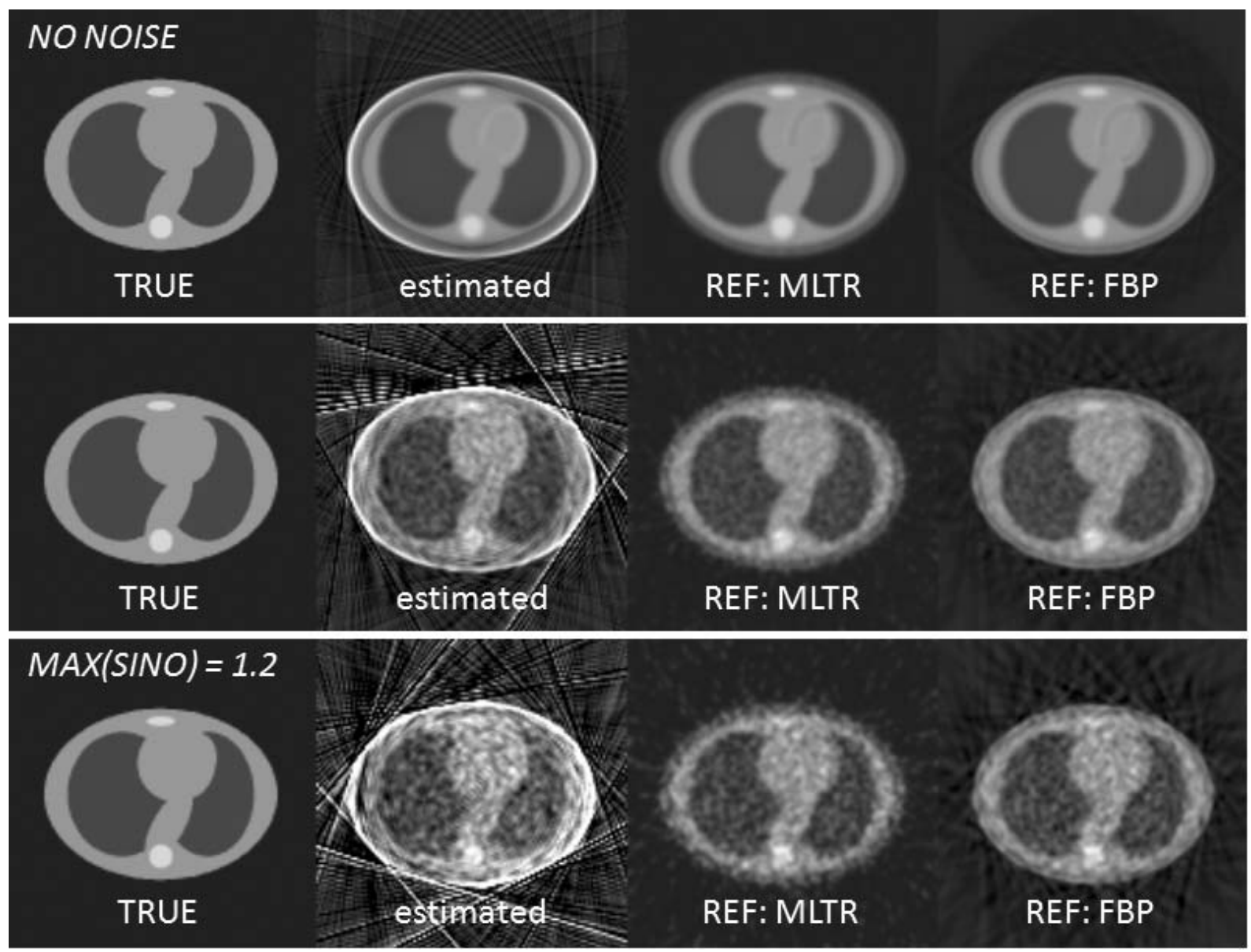

Figure 6. Reconstructions of the attenuation image from simulated data without noise (top row), with noise (middle row), and with more noise (bottom row). From left to right: the true attenuation image, the image estimated by the analytical method of section IV and FBP, and the references images reconstructed with the maximum-likelihood MLTR algorithm [6] and with FBP. These reference images were computed from the TOF-integrated attenuated emission sinogram, assuming that the true activity distribution was known.

likely that iterative algorithms will lead to significantly superior results, on the one hand by modeling the Poisson nature of the noise, and on the other hand by exploiting any available additional information on the attenuation image. It remains to be seen whether the analytic method might be useful to provide an initial estimate and thereby reduce the required number of iterations. In [29] a method based on a maximum-likelihood approach is proposed and evaluated with $2 \mathrm{D}$ simulations. The results of that study also indicate that TOF stabilizes the joint estimation problem, even in the presence of noise.

This paper deals with 2D PET data. At the end of section II, we showed how the proof of uniqueness can be extended to 3D TOF PET by applying Theorem 1 separately within a sequence of axial and transaxial planes. A direct analytical implementation of this approach is unlikely to be optimal however, and future work is ongoing to derive a $3 \mathrm{D}$ algorithm to efficiently estimate the 4D gradient of the attenuation sinogram. 


\section{Acknowledgements}

This work is supported by the grant G.0569.08 of the Fonds voor Wetenchappelijk Onderzoek Vlaanderen (F.W.O), and by a GOA-project from K.U.Leuven. The authors thank Girish Bal, Mike Casey, Maurizio Conti, Christian Michel, Vladimir Panin and Charles Watson (Siemens Healthcare, Molecular Imaging, Knoxville, TN) for useful discussions.

\section{Appendix 1}

To prove (8), use $d w(t) / d t=-t w(t) / \sigma^{2}, d \hat{u}^{\perp} / d \phi=\hat{u}$ and $d \hat{u} / d \phi=-\hat{u}^{\perp}$ to rewrite the LHS as

$$
\begin{aligned}
& \int_{-\infty}^{\infty} d l w(t-l)\left\{t \hat{u}^{\perp} \cdot \nabla f+s \hat{u} \cdot \nabla f-l \hat{u}^{\perp} \cdot \nabla f+s \frac{(t-l)}{\sigma^{2}} f-\sigma^{2} \frac{(t-l)}{\sigma^{2}} \hat{u}^{\perp} \cdot \nabla f\right\} \\
= & \int_{-\infty}^{\infty} d l w(t-l)\left\{s \hat{u} \cdot \nabla f+s \frac{(t-l)}{\sigma^{2}} f\right\}=s \int_{-\infty}^{\infty} d l \frac{d}{d l}(w(t-l) f)=0
\end{aligned}
$$

where the argument of $f$ is everywhere as in equation (1).

\section{Appendix 2}

Lemma. Consider the 2D Radon transform $p(\phi, s)$ of some non-negative smooth function $f(\vec{x})$, and two lines of response $A$ and $B$ such that $p(A) \neq 0$ and $p(B) \neq 0$. Then $A$ and $B$ can be linked by a continuous piece-wise smooth curve $(\phi(u), s(u))$ in the sinogram, parametrized by a real parameter $u \in[0,1]$ and such that $(\phi(0), s(0))=A$ and $(\phi(1), s(1))=B$, and such that $p(\phi(u), s(u))>0$ for $0 \leq u \leq 1$.

Proof. Since $p(A) \neq 0$, there is at least one point $\vec{a} \in A$ such that $f(\vec{a})>0$. Similarly, take

some point $\vec{b} \in B$ such that $f(\vec{b})>0$. The curve is defined as follows: first rotate line of response $A$ around point $\vec{a}$ until it contains both $\vec{a}$ and $\vec{b}$. Since all these lines contain $\vec{a}$, and $f(\vec{a})>0$ and $f$ is smooth, one has that $p>0$ for all these lines. Next, rotate the line of response around $\vec{b}$ until it coincides with $B$. Again, $p$ remains strictly positive during this rotation because $f(\vec{b})>0$.

\section{References}

[1] Kinahan P E, Townsend D W, Beyer T, and Sashin D 1998 Attenuation correction for a combined 3D PET/CT scanner Med Phys 25 (10) 2046-2053

[2] Carney J, Beyer T, Brasse D, Yap J T, Townsend D W 2002 CT-Based Attenuation Correction for PET/CT Scanners in the Presence of Contrast Agent. IEEE Nuclear Science Symposium and Medical Imaging Conference.

[3] Schreibmann E, Nye JA, Schuster DM, Martin DR, Votaw J, Fox T 2010 MR-based attenuation correction for hybrid PET-MR brain imaging systems using deformable image registration Med Phys 37(5) 2101-2109

[4] Keereman V, Fierens Y, Broux T, Deene Y D, Lonneux M, and Vandenberghe S 2010 MRI-based attenuation correction for PET/MRI using ultrashort echo time sequences $J$ Nucl Med 51 (5) 812818 
[5] Hofmann M, Bezrukov I, Mantlik F, Aschoff P, Steinke F, Beyer T, Pichler BJ, Schlkopf B 2011 MRIbased attenuation correction for whole-body PET/MRI: quantitative evaluation of segmentationand atlas-based methods $J$ Nucl Med 52(9) 1392-9

[6] Nuyts J, Dupont P, Stroobants S, Maes A, Mortelmans L, Suetens P 1999 Evaluation of maximumlikelihood based attenuation correction in positron emission tomography IEEE Trans Nucl Sci $\mathbf{4 6}$ (4) 1136-1141

[7] Natterer F 1987 Attenuation correction in emission tomography Inverse Problems 21-23 ed. Sabatier, Academic Press

[8] Censor Y, Gustafson D E, Lent A, Tuy H 1979 New approach to the emission computerized tomography problem: simultaneous calculation of attenuation and activity coefficients IEEE Nucl. Sc., special issue

[9] Erdogan H and Fessler J A 1999 Joint estimation of attenuation and emission images from PET scans Proc. IEEE Nucl. Sci. Symp. Med. Im. Conf. 3 1672-1675

[10] Clinthorne N H, Fessler J A, Hutchins G D, and Rogers W L 1991 Joint maximum likelihood estimation of emission and attenuation densities in PET —it Proc. IEEE Trans. Nucl. Sci. Symp. Med. Im. Conf. 3 1927-1932

[11] Glatting G, Wuchenauer M and Reske S N 2000 Simultaneous iterative reconstruction for emission and attenuation images in positron emission tomography, Med. Phys 27 (9) 2065-2071

[12] Natterer F and Herzog H 1992 Attenuation correction in positron emission tomography Mathematical Methods in the Applied Sciences 15 321-330

[13] Welch A, Campbell C, Clackdoyle R, Natterer F, Hudson M, Bromiley A, Mikecz P, Chillcot F, Dodd M, Hopwood P, Craib S, Gullberg G T and Sharp P 1998 Attenuation correction in PET using consistency information, IEEE Trans. Nuclear Science 45 (6) 3124-3141

[14] Nuyts J, Dupont P, Stroobants S, Benninck R, Mortelmans L, Suetens P 1999 Simultaneous maximum a posteriori reconstruction of attenuation and activity distributions from emission sinograms IEEE Trans Med Imag 18 393-403

[15] Krol A, Bowsher J E, Manglos S H et al 2001 An EM algorithm for estimating SPECT emission and transmission parameters from emission data only' IEEE Trans Med Imaging 20 218-232

[16] De Pierro A, Crepaldi F 2006 Activity and attenuation recovery from activity dataonly in emission computed tomography Comput Appl Math 25 2-3

[17] Bronnikov A V 2000 Reconstruction of attenuation map using discrete consistency conditions - it IEEE Trans. Medical Imaging 19(5) 451-462.

[18] Bai C, Kinahan P E, Brasse D, Comtat C, Townsend D W, Meltzer C C, Villemagne V, Charron M, and Defrise M 2003 Effects of attenuation on tumor detection in wholebody PET oncology imaging Journal of Nuclear Medicine 44 1855-1861

[19] Turkington T G, Wilson J M 2009 Attenuation artifacts and time-of-flight PET, Record Nuclear Science Symposium Conference (NSS/MIC) 2297-2299.

[20] Andre Salomon, Volkmar Schulz, Ralph Brinks, Bernd Schweizer, Andreas Goedicke and Til Aach, Iterative generation of attenuation maps in TOF-PET/MR using consistency conditions, J Nucl Med. 2009; 50 (Supplement 2):2013

[21] Conti M 2011 Why is TOF PET reconstruction a more robust method in the presence of inconsistent data? Phys Med Biol 56 155-168

[22] Salomon A, Goedicke A, Schweizer B, Aach T, Schulz V 2011 Simultaneous Reconstruction of Activity and Attenuation for PET/MR IEEE Trans Med Imag 30 804-813

[23] Panin V Y, Defrise M, Casey M E 2010 Restoration of fine azimuthal sampling of measured TOF projection data Records 2010 IEEE Nuclear Science Symposium and Medical Imaging Conference, Knoxville paper M17-4

[24] Defrise M, Panin V Y, Michel C, Casey M E 2008 Continuous and Discrete Data Rebinning in Timeof-Flight PET IEEE Trans Med Imag 27 1310-1322

[25] Mollet P, Keereman V and Vandenberghe S 2011 Experimental evaluation of simultaneous emission 
and transmission imaging using TOF information Records 2011 IEEE Nuclear Science Symposium and Medical Imaging Conference, Valencia paper M12-91

[26] Ettl S, Kaminski J, Knauer M C, and Häusler G 2008 Shape reconstruction from gradient data, Applied Optics 47 (12) , 2091-2097.

[27] Byrne C L 2008 Applied iterative methods, Wellesley, MA: AK Peters, LTd

[28] Gray R M 1972 On the asymptotic eigenvalue distribution of Toeplitz matrices IEEE Trans Information Theory IT-18 (6) 725-730

[29] Rezaei A, Nuyts J, Defrise M, Bal G, Michel C, Conti M, Watson C 2011 Simultaneous reconstruction of activity and attenuation in Time-of-flight PET Records 2011 IEEE Nuclear Science Symposium and Medical Imaging Conference, Valencia paper M8-7 